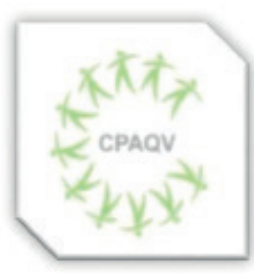

ISSN: 2178-7514

\title{
ANÁLISE DO IMPACTO DA PANDEMIA DE SARS-COV-2 SOBRE A VIDA DE PESSOAS COM DEFICIÊNCIA: REVISÃO DA LITERATURA
}

\section{Analysis of the impact of the Sars-Cov-2 pandemic on the lives of people with disabilities: literature review}

Ana Viviane Herculano Santiago Silva ${ }^{1}$; Oliver Matheus Santiago Araújo ${ }^{1}$; Renato da Costa Teixeira ${ }^{3,5}$; Soanne Chyara Soares Lira ${ }^{4,5}$

Vol. 13 | No. 3| Ano 2021

\section{RESUMO}

A pandemia de coronavírus tem sido marcada pela necessidade de intervenções a fim de amenizar a disseminação da doença e suas consequências. Essas mudanças intensificaram as barreiras enfrentadas pelas pessoas com deficiência. Essa revisão integrativa tem como objetivo analisar o impacto da pandemia na vida de pessoas com deficiência. A busca pelos dados foi realizada nas bases de dados CAPES, PubMed (MEDLINE), Scielo e PsychInfo, utilizando as seguintes combinações: Disabled Persons AND Coronavirus Infections, Disabled Persons AND Covid-19, Pessoas com Deficiência AND Infecções por Coronavirus e Pessoas com Deficiência AND covid-19. Foram selecionados sete artigos dentro dos critérios de inclusão e exclusão, para a análise na revisão integrativa, abordando diversos impactos da pandemia do novo coronavírus na vida de pessoas com deficiência. A partir da leitura e análise desses materiais, agrupou-se os impactos levantados em quatro aspectos complementares: sociais, políticos, psicológicos e biológicos, ficando evidente que a pandemia impactou negativamente este grupo, exacerbando dificuldades que já eram sua realidade cotidiana, como o entrave no acesso ao mercado de trabalho e a serviços de saúde, ressaltandose que estes fatores, aliados ao risco elevado que este grupo possui de desenvolver formas graves de Covid-19, subsidiaram o aumento de transtornos psicológicos, tais quais a síndrome do estresse pós traumático e a depressão. Desse modo, é necessário o desenvolvimento de estratégias que arrefeçam essas repercussões a curto e a longo prazo, visando garantir o respeito aos direitos humanos fundamentais.

Palavras-chave: Pessoas com deficiência; Covid-19; Qualidade de vida, Indicadores de Qualidade de Vida, SARSCoV-2.

\begin{abstract}
The coronavirus pandemic has been marked by the need for interventions to ease the spread of the disease and its consequences. These changes intensified the barriers faced by people with disabilities. This integrative review aims to analyze the impact of the pandemic on the lives of people with disabilities. The data search was performed in the CAPES, PubMed (MEDLINE), Scielo and PsychInfo databases, using the following solutions: People with disabilities and Coronavirus infections, People with disabilities E Covid-19, People with disabilities and Coronavirus infections and People with Disabilities E covid-19. Seven articles were selected within the inclusion and exclusion criteria, for analysis in the integrative review, addressing different impacts of the new coronavirus pandemic on the lives of people with disabilities. From the reading and analysis of data, the impacts raised were grouped into four complementary aspects: social, political, psychological and biological, making it evident that the pandemic negatively impacted this group, exacerbating difficulties that were already their daily reality, such as the obstacle in access to the labor market and health services, it is noteworthy that these factors, combined with the high risk that this group has of developing severe forms of Covid-19, supported the increase in psychological disorders, such as post stress syndrome. traumatic and depression. Thus, it is necessary to develop a strategy that reduces these short and long-term repercussions, while respecting fundamental human rights.
\end{abstract}

Keywords: Disabled Persons; Covid-19; Quality of Life; Indicators of Quality of Life; SARS-CoV-2.

1 Acadêmicos do curso de Fisioterapia da Universidade do Estado do Pará (UEPA)

2 Doutor em Educação pela Pontifícia Universidade Católica do Rio de Janeiro (PUC-RJ)

3 Mestre em Neurociências e Biologia Celular pela Universidade Federal do Pará (UFPA)

4 Docentes da Universidade do Estado do Pará (UEPA)

Autor de correspondência

Soanne Chyara Soares Lira - soanne.lira@uepa.br

DOI: $10.36692 / \mathrm{v} 13 \mathrm{n} 3-12 \mathrm{R}$ 


\section{INTRODUÇÃO}

A pandemia de coronavírus (COVID-19)

tem sido marcada pela necessidade de intervenções a fim de amenizar a disseminação da doença e suas graves consequências. Para o enfrentamento da disseminação do vírus, além das mudanças comportamentais da sociedade, como o distanciamento social e o uso dos equipamentos de proteção, foram utilizadas estratégias emergenciais de combate, como o fechamento de locais não essenciais e o bloqueio de cidades e estados ${ }^{(5)}$. Essas barreiras acentuam ainda mais as adversidades vivenciadas diariamente pelas pessoas com deficiência, dificultando o convívio social e o acesso aos serviços ${ }^{(1)}$.

De acordo com a Classificação Internacional de Funcionalidade, Incapacidade e Saúde, o termo deficiência é definido como "problemas na função ou estrutura corporal, tais como um desvio ou perda significativa"(10); e inclui limitações de atividades e restrições de participação, considerando a interação do indivíduo e o ambiente. A deficiência, para a Organização Mundial da Saúde, é um problema de saúde pública global, devido às suas consequências socioeconômicas, como as discriminações e desigualdades sofridas ao longo da vida; e o aumento de gastos, devido às necessidades de atendimento de saúde ${ }^{(21)}$.

Desde a infância, pessoas com deficiência (PCD) são expostas às desigualdades, tendo oportunidades limitadas comparadas às crianças sem deficiências, e um ambiente social afetado, tendo em vista que os familiares desses indivíduos, possuem maior taxa de perda de trabalho, além de altos custos necessários para o bem estar geral $^{(6)}$. No atual contexto, esses obstáculos foram intensificados, afetando a qualidade de vida em PCD, a exemplo da acentuação de ansiedade, depressão, pensamentos suicidas e insônia ${ }^{(9)}$; ou mesmo de dificuldades para o acesso aos serviços essenciais $^{(2)}$.

A qualidade de vida também foi afetada por barreiras, como a dificuldade para o acesso a serviços, no geral; obstáculos nas ruas e calçadas, e déficit de acessibilidade nos transportes públicos $^{(13)}$. Além disso, nota-se que algumas ferramentas que surgiram de forma benéfica, e com função imprescindível para o combate ao COVID-19, como o uso dos equipamentos de proteção individuais e o acompanhamento remoto da saúde, tornaram -se obstáculos que afetam, de forma não intencional, a qualidade de vida e interação social, dificultando o acesso às informações, e favorecendo consequências psicológicas $^{(11)}$. A assistência e monitoramento da saúde, de modo remoto, por exemplo, pode ser um desafio para PCD, especialmente àquelas com comprometimento auditivo, que demandam da língua de sinais, para a compreensão adequada das informações do profissional da saúde ${ }^{(15)}$.

O uso dos equipamentos de proteção individuais, como as máscaras faciais, oculta as expressões faciais e dificulta a leitura labial: fatores importantes para a comunicabilidade 
de PCD. Aqueles com comprometimento visual, têm a sua qualidade de vida e acesso aos serviços, também comprometidos, considerando a necessidade do contato frequente com objetos públicos para se locomover, assim como auxílio de diferentes pessoas para garantir as atividades diárias, em decorrência da falta de acessibilidade e adaptações em lugares públicos ${ }^{(7)}$.

Como consequência das intervenções e barreiras promovidas pela pandemia e pela necessidade de isolamento social, notam-se efeitos psicossociais na vida de PCD. O ambiente estressante, decorrente das incertezas promovidas pela pandemia, de mudanças repentinas na rotina, limitações financeiras e ausência de recursos e espaços seguros para manifestar as violências sofridas, agravam a vulnerabilidade frente à violência de $\operatorname{PCD}^{(14)}$. Ainda nesse contexto, os indivíduos que precisam de auxílio para a execução de atividades, enfrentam uma maior dependência em relação aos cuidadores ou familiares, aumentando ainda mais a possibilidade de violência ${ }^{(8)}$; e outra consequência na qualidade de vida e funcionalidade de PCD, é a alta probabilidade, até 4 vezes mais, de PCD que vivem na cidade durante a pandemia, lesionarem -se, e morrerem comparadas a pessoas sem deficiência ${ }^{(13)}$.

É necessário que surjam melhorias no acesso global aos serviços, com sistemas de saúde inclusivos e acessíveis, e políticas de saúde que considerem as necessidades das pessoas com deficiência. Assim sendo, diante da necessidade de estudos acerca da pandemia de COVID-19 e pessoas com deficiência, o objetivo desse estudo é analisar o impacto da pandemia na vida de pessoas com deficiência.

\section{MATERIAIS E MÉTODOS}

Este estudo é uma revisão integrativa, dedicada a catalogar e interpretar as pesquisas científicas disponíveis na literatura, sobre impactos da pandemia do Sars-Cov-2 na vida de pessoas com deficiência. Desde a sua concepção à publicação, foram estabelecidas seis etapas metodológicas: delimitação da tônica do estudo e definição da questão norteadora da pesquisa, eleição dos fatores de inclusão e exclusão de estudos/busca na literatura especializada, tabulação dos dados coletados, avaliação dos estudos incluídos na revisão, interpretação dos resultados e apresentação da revisão/síntese da versão final do estudo ${ }^{(12,19)}$.

$\mathrm{Na}$ primeira fase, deste modo, foi definida a questão norteadora: "Qual o impacto da pandemia do Sars-Cov-2 na vida da pessoa com deficiência física?’. Para a seleção da literatura, foram adotados os seguintes critérios de inclusão: estudos que abordem sobre a deficiência física e covid-19, estudos clínicos, opiniões, narrativas, editoriais, revisões não sistemáticas ou não integrativas, disponíveis para acesso online, gratuito, na íntegra e publicados entre dezembro de 2019 e março 2021. Foram considerados critérios de exclusão: estudos duplicados; revisões 
sistemáticas ou integrativas e, cujo escopo não contivesse descrição associada à discussão/ análise, delimitada previamente por esta pesquisa. Estimando a qualidade dos dados obtidos, foram selecionadas como bases de dados do portal de periódicos da Coordenação de Aperfeiçoamento de Pessoal de Nível Superior (CAPES), a PubMed da Medical Literature Analysis and Retrieval System Online (MEDLINE), a Scientific Electronic Library Online (Scielo) e o PsycInfo (FIGURA 1). Para a realização da busca, foi realizada a combinação dos operadores booleanos por meio dos conectores "AND” (combinação restritiva), tendo sido utilizadas as seguintes combinações: Disabled Persons AND Coronavirus Infections, Disabled Persons AND Covid-19, Pessoas com Deficiência AND Infecções por Coronavirus, Pessoas com Deficiência AND covid-19.

A busca de dados foi realizada por dois pesquisadores, de forma independente, visando analisar a aplicabilidade prática da metodologia de busca nas bases de dados selecionadas. Foram analisados os títulos e o resumo das publicações encontradas seguindo os fatores de inclusão e exclusão, previamente citados; sendo estipulado que, em caso de discordância, um terceiro pesquisador decidirá se o documento seria integrado ou não à pesquisa.

\section{RESULTADOS}

A busca realizada nas bases de dados selecionadas, identificou 45 artigos (FIGURA 1), sendo que destes, 24 foram encontrados na plataforma CAPES, 11 na PUBMED e 11 no PsycInfo; no SCIELO não foram encontrados resultados envolvendo os operadores booleanos e os termos selecionados. Dentre os artigos encontrados, 5 artigos foram eliminados por estarem duplicados, 32 foram excluídos porque na leitura do título e do resumo ficou claro que não tratavam sobre os impactos da pandemia associados aos deficientes físicos, e apenas um foi excluído pela leitura integral do texto realizada individualmente por dois pesquisadores, tendo sido efetuada por se tratar de um estudo do tipo revisão de literatura, o que não contempla o escopo desta pesquisa. Deste modo, foram catalogados 7 artigos para a realização desta revisão da literatura, incluindo excepcionalmente, uma revisão que se assemelha a opiniões/ narrativas e editoriais, que também foram incluídos.

$\mathrm{Na}$ Figura 1, é possível verificar as informações referentes a cada etapa de obtenção de dados, em forma de fluxograma, estruturada em quase fases: identificação, seleção, elegibilidade, e por fim, a inclusão, de acordo com as recomendações propostas pela ferramenta Preferred Reporting Items for Systematic Reviews and Meta-Analyses (PRISMA). No Quadro 1 está exposta a distribuição dos estudos, selecionados de acordo com título, autores/ano, tipo de comunicação e os principais aspectos discutidos. 
Figura 1 - Fluxograma PRISMA dos artigos encontrados nas bases de dados CAPES, PUBMED, SCIELO E PSYCINFO. BELÉM, PA, BRASIL, 2021.

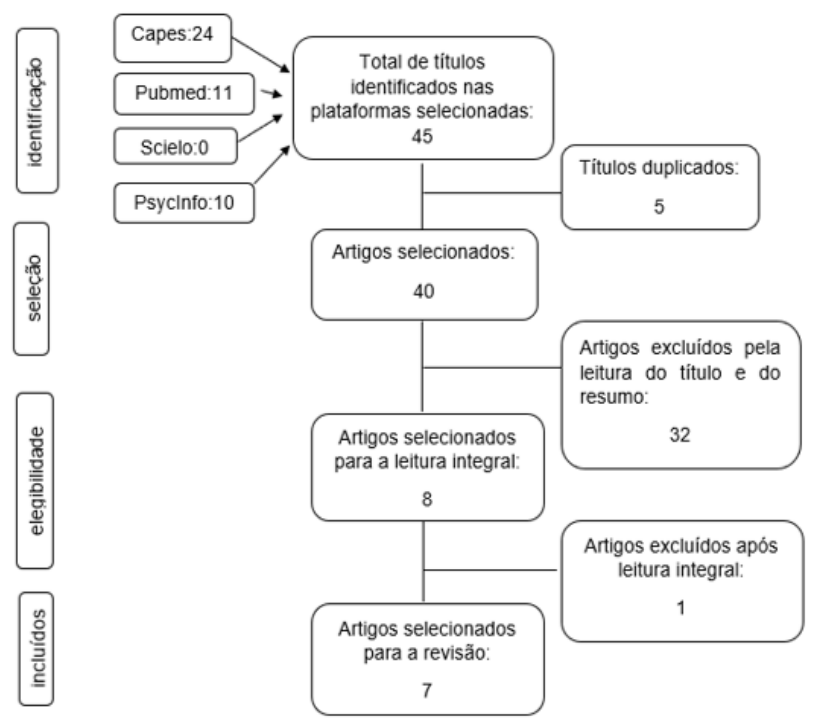

Fonte: Dados da pesquisa, 2021.

Quadro 1 - Distribuiçẫo dos estudos, selecionados de acordo com título, autores, ano, tipo de comunicaçẫo e os principais aspectos discutidos. Belém, PA, Brasil, 2021.

\begin{tabular}{|c|c|c|c|}
\hline Título & Autoresiano & $\begin{array}{l}\text { Tipo de } \\
\text { comunicaçẫo }\end{array}$ & Principais aspectos discutidos \\
\hline $\begin{array}{ll}\text { Examining the impact of } \\
\text { CoviD-19 on Stress and } \\
\text { Coping } & \text { Strategies in } \\
\text { individuais } & \text { With Disabilities } \\
\text { andChronic Condition }\end{array}$ & UMUCU; LEE, 2020. & $\begin{array}{l}\text { Estudo } \\
\text { transversal } \\
\text { Observacional }\end{array}$ & $\begin{array}{l}\text { O enfrentamento ao COVID-19 afeta o aspecto } \\
\text { psicológico e o aspecto social. Diversas } \\
\text { estratégias podem ser adotadas para contornar } \\
\text { essa situaçấo, entre elas a distraçấo e aceitação. }\end{array}$ \\
\hline $\begin{array}{l}\text { Deafness and Mental Heaith: } \\
\text { Cinical Chalienges During the } \\
\text { CoviD-19 Pandemic }\end{array}$ & $\begin{array}{l}\text { RECIO-BARBERO; } \\
\text { SAENZ-HERRERO; } \\
\text { SEGARRA, } 2020\end{array}$ & Comentário & $\begin{array}{l}\text { A comunidade surda enfrenta barreiras que } \\
\text { afetam o seu bem-estar geral, mas principalmente } \\
\text { os aspectospsicológicose sociais. }\end{array}$ \\
\hline $\begin{array}{l}\text { Reflexốes sobre a pandemia } \\
\text { da CoviD-19 e pessoas com } \\
\text { deficiência }\end{array}$ & $\begin{array}{l}\text { COURA; ALMEIDA, } \\
2020 .\end{array}$ & Editorial & $\begin{array}{l}\text { As pessoas com deficiência enfrentam barreiras } \\
\text { adicionais durante a pandemia da COVID-19, } \\
\text { incluindo as relacionadas com os aspectos sociais } \\
\text { e políticos, intensificando as situaçốes de } \\
\text { vulnerabilidade. }\end{array}$ \\
\hline $\begin{array}{l}\text { Disability inclusiveness of } \\
\text { government responses to } \\
\text { CoviD-19 in South America: a } \\
\text { frameworkanalysis study }\end{array}$ & $\begin{array}{l}\text { SAKELLARIOU; } \\
\text { MALFITANO; } \\
\text { ROTAROU, } 2020 .\end{array}$ & $\begin{array}{l}\text { Análise } \\
\text { documental }\end{array}$ & $\begin{array}{l}\text { Pessoas com deficiência sẫo mais expostas aos } \\
\text { efeitos da pandemia na condiçẫo socioeconômica, } \\
\text { política e em relaçấo ao acesso aos serviços. }\end{array}$ \\
\hline $\begin{array}{l}\text { Narrativas de sujeitos com } \\
\text { deficiência e isolamento social } \\
\text { em tempos de pandemia }\end{array}$ & $\begin{array}{l}\text { COSTA; } \quad \text { BATISTA; } \\
\text { TAVARES, } 2020 .\end{array}$ & Narrativa & $\begin{array}{l}\text { Em uma perspectiva sociológica, as narrativas } \\
\text { biográficas, e também autobiográficas tendo em } \\
\text { vista que os três autores do manuscrito sâo } \\
\text { pessoascom deficiênciavisual, acentua se queos } \\
\text { contextos mais afetados sấo o psicológico e o } \\
\text { social. }\end{array}$ \\
\hline $\begin{array}{l}\text { impact of CoviDis pandemic } \\
\text { on peopie iving with visual } \\
\text { disability }\end{array}$ & SEN.JAM, 2021 & $\begin{array}{l}\text { Análise } \\
\text { bibliográfica }\end{array}$ & $\begin{array}{l}\text { Pessoas com deficiência sã̃o maisvulneráveis a } \\
\text { contraírem a severa infeç̧ẫo de Sars-cov-2, que } \\
\text { pessoas sem deficiência; os aspectos biológicos, } \\
\text { psicológicose sociais sấo os mais afetados. }\end{array}$ \\
\hline $\begin{array}{l}\text { Disabied people in the time of } \\
\text { coviD-19: identifying needs, } \\
\text { promoting inciusivity }\end{array}$ & $\begin{array}{l}\text { ROTAROU; } \\
\text { SAKELLARIOU; } \\
\text { KAKOULLIS; } \\
\text { WARREN, } 2021 .\end{array}$ & Comentário & $\begin{array}{l}\text { A pandemia tornou mais graves as carências que } \\
\text { já existiam na vida de pessoas com deficiência, } \\
\text { como o acesso aos serviços de saúde, ao } \\
\text { mercado de trabalho, à educaçẫo e à renda, fator } \\
\text { que representaria a falência do Estado emgarantir } \\
\text { políticas públicas adequadas a este público, } \\
\text { ressaltando os aspectos biológicos, sociais e } \\
\text { políticos. }\end{array}$ \\
\hline
\end{tabular}

Fonte: Dados da pesquisa, 2021 


\section{DISCUSSÃO}

Os resultados apresentados pelos estudos selecionados, estabelecem intrínseca relação entre as múltiplas peculiaridades que as pessoas com deficiência visual, auditiva, motora e intelectual, possam vir a possuírem; e o contexto da pandemia, gerada pelo novo Coronavírus. A questão norteadora para esta discussão será “Qual o impacto da pandemia do Sars-Cov-2 na vida da pessoa com deficiência?". Antes de delinear os resultados, é fundamental pontuar que tal pesquisa foi realizada em um período da história humana contemporânea, completamente singular, e, portanto, este ineditismo implica a não catalogação de estudos do tipo experimental, sendo a revisão constituída então, por estudos do tipo análise documental e estudo transversal observacional, além de editorias, narrativas, comentários e uma análise bibliográfica.

Outro fator que fundamenta a importância da realização deste estudo, é o fato de que durante a coleta de dados, não foi encontrado nenhum resultado envolvendo os descritores Disabled Persons AND Coronavirus Infections, Disabled Persons AND Covid-19, Pessoas com Deficiência AND Infecções por Coronavirus, Pessoas com Deficiência AND covid-19 na plataforma SCIELO, portal no qual, por se tratar de uma referência extremamente popular no meio científico e na academia, esperava-se encontrar artigos envolvendo a análise desta parcela tão significativa da população global.

\section{Os principais impactos elencados}

Define-se que a partir da literatura catalogada e interpretada nesta revisão, serão evidenciados, sobremaneira, os aspectos que englobam a qualidade de vida e o bem-estar, ressaltando principalmente as repercussões da pandemia de Sars-Cov-2 em quatro diferentes, porém complementares, áreas:

- No aspecto da vida social, em suas dimensões econômica, segurança pessoal e na estruturação antropológica da sociedade(17, 20, 16, 4);

Nas limitações do exercício cidadão de direitos politicamente assegurados, como o acesso à saúde e à livre circulação, e como diferentes governos atuaram na intenção de conter estes; e os demais reflexos políticos da pandemia $(16,4,15,18)$;

- $\quad \mathrm{Na}$ dimensão da saúde mental, e as formas com as quais as pessoas com deficiência, mitigaram os impactos psicológicos gerados ou exacerbados pela pandemia $(20,18$, $3,15)$

- Nos aspectos biológicos da manutenção da saúde física, com a necessidade de controle biológico de infecções e de diminuição de agravos em saúde(4, 20, 16, 17, 18). 
Os principais impactos no aspecto social

No que tange à esfera social, fica evidente que as condições historicamente estabelecidas de marginalização e discriminação de pessoas com deficiência, tornaram-se mais complexas durante o período pandêmico, acentuando a ineficiência do Estado em promover a equidade social, sem estabelecer um discurso de vulnerabilidade capacitista ${ }^{(17)}$, muito comumente adotado para oprimir este grupo. Desse modo, pessoas com deficiência que não recebam o adequado suporte, serão negativamente impactadas pelos efeitos da pandemia de forma desproporcional, se comparados com pessoas sem condições crônicas de saúde ou sem deficiência (20).

Outrossim, segmentos específicos dentro do grupo das pessoas com deficiência, ainda encontram maior índice de risco para outros agravantes do bem-estar social, onde vemos a questão que se refere ao aumento de violência entre pessoas com deficiência em diferentes contextos, como crianças, pessoas privadas de liberdade, pessoas em situações de rua e em condição de emergência humanitária $(16,17)$. Pontua-se também que pessoas com deficiência do sexo feminino, ainda têm que lidar com os impactos da misoginia e do feminicídio, instaurado nas culturas patriarcais, sendo esta violência de gênero, mais uma barreira enfrentada em suas vidas no presente cotidiano ${ }^{(16,17)}$.
Ademais, outra vertente social abalada, foi o aspecto econômico ${ }^{(4)}$, influenciando de forma negativa, no acesso à renda e ao mercado de trabalho, inegável fator da subsistência digna para o grupo familiar da PCD e para a dinâmica econômica regional, podendo estar diretamente associado à perversa inclusão de vários indivíduos na linha da pobreza ou abaixo dela, já que pessoas com deficiência vivenciam níveis inferiores de escolarização e empregabilidade, se comparadas a pessoas sem deficiência $^{(16)}$.

\section{Os principais impactos no aspecto} político

O exercício político dos direitos e deveres da PCD, foram igualmente afetados negativamente ao longo da crise sanitária internacional, fazendo com que fatores básicos para a vida humana, como o acesso a serviços de saúde $(16,4)$ e o direito de ir e vir, tenham sido limitados, quer seja pelo entrave na instauração de comunicação com os profissionais de saúde, devido à impossibilidade da realização da leitura labial pelo uso de máscaras de proteção individual, muito frequentemente relatada por indivíduos com surdez ${ }^{(17)}$, quer seja pela dificuldade de locomoção sem a assistência de cuidadores, condição afetada neste cenário onde o recomendado é a manutenção de distanciamento social ${ }^{(4,18)}$.

Outra importante condição a ser avaliada na esfera política, é a multiplicidade 
de espaços geopolíticos dispostos ao redor do mundo, sendo cada território dotado de condições culturais e sociais próprias que, dentro de suas limitações e potencialidades, encontraram subterfúgios para lidar com a situação imposta, ressaltando-se que, nações que tradicionalmente já viviam em vulnerabilidade econômica e desigualdade de classes, encontraram ainda maior discrepância entre as medidas que o Governo estabeleceu como necessárias, e a sua execução de fato ${ }^{(18,17)}$.

Em países da América do Sul, os indivíduos com deficiência não foram completamente respeitados pelo primeiro setor, tendo o seu risco de pobreza multidimensionalmente aumentado, primordialmente pelo fato de este grupo possui, majoritariamente, empregos precários, não especializados ou com baixa remuneração e, secundariamente, pelo registro incompleto destas pessoas em órgãos do governo, que prestem assistência social ${ }^{(17)}$.

Não obstante, nações com maior Índice de Desenvolvimento Humano (IDH), como por exemplo, as localizadas no continente europeu, similarmente enfrentaram dificuldades para tornar universalizados serviços que inevitavelmente tiveram de ser adaptados a esta nova realidade, como ferramentas online de suporte em saúde(15).
Principais impactos no aspecto psicológico

Dentro da dimensão psicológica, já se comprovou que pessoas com deficiência possuem maiores níveis de percepção do estresse, sentido quando existe a concomitante degradação da saúde física e da qualidade de vida(4), destarte, todas as referências utilizadas foram unânimes em pontuar que a pandemia gerou ampla gama de alterações psíquicas nas pessoas com deficiência. A alteração de humor, o aumento significativo da ansiedade, da depressão, do estresse, do medo e a síndrome do estresse pós-traumático foram as condições de saúde mental mais frequentemente retratadas entre este grupo ${ }^{(4,18)}$.

Neste ínterim, as condições de saúde mental descritas, estão muito atreladas ao contato assíduo com notícias ruins, ao distanciamento do convívio diário com amigos e familiares, e também à alteração repentina das atividades de vida laboral e da rotina(3); acrescenta-se que uma importante contribuição para a interpretação desta realidade, é analisar o ponto de vista de uma pessoa com deficiência sobre a própria condição de saúde mental neste contexto:

Eu tinha ansiedade e crise de pânico, e depois da pandemia piorou e aumentou. Meu cotidiano já não era tão fácil, por ter sido diagnosticada com crise de ansiedade e pânico, bem como agora percebo que estou sofrendo com os noticiários televisionados e redes sociais; confesso que a imprevisibilidade de tempo presente e futuro, causam aumento significativo das crises que foram mencionadas anteriormente ${ }^{(3)}$. 
Em vista disso, não somente medidas que fornecem suporte emocional já estabelecidas de forma comunitária, como o lazer e a religião, foram adotados por estas pessoas para contornar esta fase de inigualável dificuldade, mas também as mídias sociais, aplicativos adaptados e os meios de comunicação se destacaram como ferramentas de apoio ao sofrimento mental, tendo em vista que os serviços de saúde pública, não têm conseguido contemplar as demandas da população ${ }^{(20,15,3)}$.

Em contrapartida, outros autores enfatizam que devido à necessidade da realização de um distanciamento social, com o objetivo de mitigar a disseminação da Covid-19, prejudicou-se de forma veemente a interação social desta população, e, mesmo no atual contexto onde as redes sociais e demais aplicativos de smartphone são plataformas que podem ser muito úteis ${ }^{(18)}$, muitos destes não são completamente acessíveis ou ainda representam uma lacuna no mercado, quando se trata de pessoas com restrição de comunicação, como é o caso das pessoas com surdez total ou parcial, e das pessoas cegueira total ou parcial $(15,18)$.

\section{Principais impactos no aspecto}

\section{biológico}

Como já levantado, a pandemia afetou de forma mais incisiva, grupos mais socialmente marginalizados. Dentro desse contexto, os indicadores em saúde salientam que pessoas que convivem com condições crônicas de saúde, e pessoas com deficiência, possuem risco maior de contaminação e de desenvolvimento de casos mais graves de Covid-19(20,16,17).

O estado de exceção, gerado pela conjuntura de infecção massiva pelo COVID-19, suscitou a instauração de medidas de controle sanitário em escala internacional, como a higienização adequada das mãos, o uso de equipamentos de proteção individual e a etiqueta respiratória ${ }^{(4)}$; no entanto muitas pessoas desta população vivem em condições precárias de vida, e nem mesmo têm condição de acesso à água tratada, aumentando ainda mais a vulnerabilidade do dano à integridade da saúde ${ }^{(16)}$.

Não obstante, a não disponibilidade de informação segura, adaptada e de fácil compreensão ao acesso da população de PCDs, principalmente as pessoas com deficiência intelectual e os grupos que precisam de comunicação adaptada, como é o caso dos deficientes auditivos e visuais, acaba por se tornar outro agravante para o combate ao SarsCov-2 $2^{(4,18)}$.

As pessoas com deficiência visual, ainda encontram outra barreira, que é a necessidade do toque para realizar a leitura, estando muito mais susceptíveis ao contato com superfícies contaminadas, reintera-se que pessoas com cegueira total ou parcial, que moram em zona rural, correm ainda maiores riscos de infecção, que pessoas sem esta deficiência que habitem a 
mesma área ${ }^{(18,4)}$.

\section{CONCLUSÃO}

O desenvolvimento da presente pesquisa, partiu da percepção de que há uma lacuna na literatura científica, sobre a análise das repercussões do maior evento de saúde pública da história humana recente, na vida deste grupo de pessoas socialmente invisibilizadas, e politicamente ignoradas, que é o das pessoas com deficiência; e o diminuto acervo catalogado acabou por afirmar esta assertiva, quer seja por sua quantidade, quer seja pelas informações nele obtidas.

O objetivo deste estudo foi analisar os impactos da pandemia de Sars-Cov-2 na vida da PCD, e conclui-se que este cenário hodierno impactou negativamente a qualidade de vida desse público dentro dos aspectos sociais, políticos, psicológicos e biológicos.

Remontar períodos pregressos da história da humanidade, auxilia na percepção do quanto o capacitismo e outros aparatos, utilizados para o controle social e para a segregação, ainda hoje exercem influência sobre a vida dos socialmente marginalizados e das maiorias minorizadas. A pandemia se manifestou de forma diversa nas nações, gerando políticas públicas e intervenções igualmente diversas, como é próprio da adaptabilidade humana; no entanto, é inaceitável que o corpo social mantenha ainda desamparada esta importante parcela de cidadãos.

O recorte temporal pandêmico, não apenas acentuou as disparidades socioeconômicas já existentes, quando se trata da parcela da população que possui deficiência, como trouxe luz a temas pouco discutidos, como o preconceito estrutural. Houve ainda a insurgência de um colapso da saúde mental e de muitos direitos estabelecidos na forma da constituição, tornando ainda pior a qualidade de vida deste grupo.

Todos os estudos apontaram níveis anormais de sensações descritas como: pânico, angústia, medo ou preocupação. A lembrança de como o mundo era antes deste episódio pandêmico, no entanto, reforça a tese de que a vida destas pessoas nunca foi permeada por facilidades, e a deterioração das mínimas condições de vida, a ampliação da ameaça de violência interpessoal e o cerceamento da cidadania, vieram agravar ainda mais condição.

Entre a gama de deficiências avaliadas, nota-se que pessoas com cegueira total ou parcial, pessoas com surdez e pessoas com deficiência no desenvolvimento intelectual e da função cognitiva, mostraram-se mais susceptíveis ao contágio pela COVID-19, sobretudo pela forma com a qual interagem com o mundo, e pela dificuldade em acessar informações seguras de prevenção de agravos e, quando adquiridas, executá-las a contento. Pessoas que precisam de auxílio de tutores ou cuidadores por sua vez, destacaram se como os mais prejudicados em suas atividades que 
demandam a saída do confinamento e o acesso a serviços.

O estudo ressalta aqui a importância de se pesquisar acerca dos parâmetros de qualidade de vida e bem-estar de PCDs, sendo estes dados fundamentais para compreender os anseios desta comunidade, e estabelecer metodologias para que estes sejam atendidos integral e humanitariamente. De posse de informações como estas, muitas medidas possivelmente arrefeceriam as repercussões da pandemia na vida da PCD em todos os aspectos, como por exemplo: programas sociais, com suporte financeiro assistencial; assistência à saúde, com equipamento multifuncional, especializada nas particularidades deste grupo, e com a utilização de tecnologia, como plataformas de teleatendimento, e acesso à educação, visando também o cenário pós pandemia, o que traria melhores possibilidades de ascensão social e o controle de desigualdades sociais.

Desta feita, é substancial que um quantitativo maior de pesquisas seja desenvolvido, visando avaliar não apenas variáveis de saúde física e mental, mas também os aspectos antropológicos e sociológicos, com o intuito de traçar metas exequíveis para lidar com as atuais e com as futuras repercussões que a pandemia da COVID-19 tenha estabelecido na vida das pessoas com variados tipos de deficiência.

\section{REFERÊNCIAS}

1. Annaswamy TM, Verduzco-Gutierrez M, Frieden L. Telemedicine barriers and challenges for persons with disabilities: COVID-19 and beyond. Disabil Health J. 2020 Oct;13(4):100973. doi: 10.1016/j. dhjo.2020.100973. Epub 2020 Jul 9. PMID: 32703737; PMCID: PMC7346769.

2. Armitage R, Nellums LB. The COVID-19 response must be disability inclusive. Lancet Public Health. 2020 May;5(5):e257. doi: 10.1016/S24682667(20)30076-1. Epub 2020 Mar 27. PMID: 32224295; PMCID: PMC7270835.

3. Costa VB, Batista CP, Tavares ERB. Narrativas de sujeitos com deficiência e isolamento social em tempos de pandemia. Rev. Ibe. Est. Ed. 2020 Out; 15(esp3):247189.

4. Coura AS, Almeida IJS. Reflexões sobre a pandemia da covid-19 e pessoas com deficiência. Journal health npeps. 2020 Set; 5(2): 16-19.

5. Ferguson NM, Laydon D, Nedjati-Gilani G, Imai N, Ainslie K, Baguelin M et al. Report 9: impact of nonpharmaceutical interventions (npis) to reduce covid-19 mortality and healthcare demand. Imperial college covid-19 responde team, 2020 Mar.

6. Houtrow A, Harris D, Molinero A, LevinDecanini T, Robichaud C. Children with disabilities in the United States and the COVID-19 pandemic. J Pediatr Rehabil Med. 2020;13(3):415-424. doi: 10.3233/PRM200769. PMID: 33185616.

7. Jalali M, Shahabi S, Lankarani KB, Kamali M, Mojgani P. Covid-19 and disabled people: perspectives from iran. Disability \& society, 2020 April; 35(9): 844-847.

8. Lund EM. Interpersonal violence against people with disabilities: Additional concerns and considerations in the COVID-19 pandemic. Rehabil Psychol. 2020 Aug;65(3):199-205. doi: 10.1037/rep0000347. PMID: 32804531 .

9. Necho M, Birkie M, Gelaye H, Beyene A, Belete A, Tsehay M. Depression, anxiety symptoms, Insomnia, and coping during the COVID-19 pandemic period among individuals living with disabilities in Ethiopia, 2020. PLoS One. 2020 Dec 30;15(12):e0244530. doi: 10.1371/journal. pone.0244530. PMID: 33378397; PMCID: PMC7773255.

10. Nubila HBV, Buchalla CM. O papel das classificações da oms - cid e cif nas definições de deficiência e incapacidade. Revista Brasileira de Epidemiologia, 2008; 11(2): 324-335.

11. Park J. Unraveling the Invisible but Harmful Impact of COVID-19 on Deaf Older Adults and Older Adults with Hearing Loss. J Gerontol Soc Work. 2020 Aug-Oct;63(6-7):598-601. doi: 10.1080/01634372.2020.1799282. Epub 2020 Aug 3. PMID: 32744477. 
12. Paul J, Criado AR. The art of writing literature review: what do we know and what do we need to know? . International business review, 2020 Aug; 29(4). https:// doi.org/10.1016/j.ibusrev.2020.101717.

13. Pineda VS, Corburn J. Disability, Urban Health Equity, and the Coronavirus Pandemic: Promoting Cities for All. J Urban Health. 2020 Jun;97(3):336-341. doi: 10.1007/s11524-020-00437-7. Erratum in: J Urban Health. 2021 Apr;98(2):308. PMID: 32328866; PMCID: PMC7179953.

14. Platt VB, Guedert JM, Coelho EBS. Violência contra crianças e adolescentes: notificações e alerta em tempos de pandemia. Revista Paulista de Pediatria, 2021 Jan; 39(2020267).

15. Recio-Barbero M, Sáenz-Herrero M, Segarra R. Deafness and mental health: Clinical challenges during the COVID-19 pandemic. Psychol Trauma. 2020 Aug;12(S1):S212-S213. doi: 10.1037/tra0000729. Epub 2020 Jun 1. PMID: 32478542.

16. Rotarou ES, Sakellariou D, Kakoullis EJ, Warren N. Disabled people in the time of COVID-19: identifying needs, promoting inclusivity. J Glob Health. 2021 Jan; 11:03007. doi: 10.7189/jogh.11.03007. PMID: 33643617; PMCID: PMC7897447.

17. Sakellariou D, Malfitano APS, Rotarou ES. "Disability inclusiveness of government responses to covid-19 in south america: a framework analysis study." International journal for equity in health, 2020 Aug; 19 (131). doi:10.1186/s12939-020-01244-x

18. Senjam SS. "Impact of covid-19 pandemic on people living with visual disability." Indian journal of ophthalmology, 2020; 68(7): 1367-1370. doi:10.4103/ijo. ijo_1513_20.

19. Souza MT, Silva MD, Carvalho R. Revisão integrativa: o que é e como fazer integrative review: what is it? how to do it?. Einstein. 2010; 8(1 pt 1):102-6.

20. Umucu E, Lee B. "Examining the impact of covid-19 on stress and coping strategies in individuals with disabilities and chronic conditions." Rehabilitation psychology, 2020; 65(3):193-198. doi:10.1037/rep0000328

21. Organização das Nações Unidas. Who global disability action plan 2014-2021. better health for all people with disability. Who library cataloguing, 2015.

OBSERVAÇÕES: Esta pesquisa foi financiada pela Fundação Amazônia de Amparo a Estudos e Pesquisas (FAPESPA).

Os autores declaram não existir conflitos de interesse de qualquer natureza. 